\title{
A low-grade chondrosarcoma presenting as an unusual cervical mass in the hyoid bone: a case report
}

\author{
Hirohiko Tachino, Hiroaki Fushiki", Masayuki Ishida and Yukio Watanabe
}

\begin{abstract}
Introduction: A chondrosarcoma originating from the hyoid bone is very rare. Here, we describe a case of lowgrade chondrosarcoma of hyoid origin and discuss its preoperative imaging features, including those on positron emission tomography-computed tomography, and its recurrence rate.

Case presentation: A 42-year-old Japanese man noticed a mass in the right submandibular region of his neck. A hard $3.0 \times 2.8 \mathrm{~cm}$ tumor was noted on the right side of his hyoid bone. The mass was immobile and moved with deglutition.

Conclusion: Even though radiographic studies, including positron emission tomography-computed tomography, were inconclusive, the cartilaginous tumor was surgically removed en bloc, and the tumor was diagnosed based on the results of pathological investigations. Close follow-up is recommended in such cases due to the potential for recurrences, because local recurrence occurred in $50 \%$ of the reported cases of grade one chondrosarcomas.
\end{abstract}

\section{Introduction}

Chondrosarcoma, a malignant tumor characterized by the production of a cartilage matrix, accounts for about $11 \%$ cases of primary malignant bone tumors [1]. The parts of the body where it develops at relatively high incidences include the long bones, the pelvis and the ribs [1]. The head and neck area are seldom affected; chondrosarcomas in this area reportedly account for $1 \%$ to $12 \%$ of all reported cases of the disease [1]. Chondrosarcoma of the hyoid bone is extremely rare, with only 15 cases being reported in international literature. Here, we describe a case of low-grade chondrosarcoma of hyoid origin. We also discuss its preoperative imaging features and its recurrence rate.

\section{Case presentation}

A 42-year-old Japanese man noticed a mass in the right submandibular region of his neck in June 2010. He visited the department of otolaryngology at a nearby general hospital the next month and was referred for medical treatment. More than two weeks later, he visited our

\footnotetext{
* Correspondence: hfushiki@med.u-toyama.ac.jp

Department of Otolaryngology, Head and Neck Surgery, University of Toyama, Toyama, Japan
}

University Hospital. A hard $3.0 \times 2.8 \mathrm{~cm}$ tumor was noted on the right side of his hyoid bone. The mass was immobile and moved with deglutition. No spontaneous or tender pain was noted.

Laryngeal endoscopy revealed no contributory abnormal findings. Computed tomography (CT) demonstrated a gourd-shaped distension of bone in the area extending from the body of the hyoid bone to the right greater horn. A multilocular cystic area of low density was observed inside the distension, and central ossification was evident in the anterior lesion (Figure 1a). Magnetic resonance imaging (MRI) of his neck revealed a gourdshaped nodular lesion of $2.5 \times 2.2 \mathrm{~cm}$ (upper part) and $1.6 \times 1.0 \mathrm{~cm}$ (lower part) on the right side of his hyoid bone. The signal intensity was low in T1-weighted images and high in T2-weighted images, with a mosaic pattern being observed in the high signals (Figure 1b, c). Based on these findings, a cartilaginous tumor originating from the right side of his hyoid bone was diagnosed. No abnormal accumulation was detected by bone scintigraphy or fluorodeoxyglucose positron emission tomography (FDG-PET) (Figure 2). Therefore, high-grade malignant chondrosarcoma was diagnosed by exclusion. 


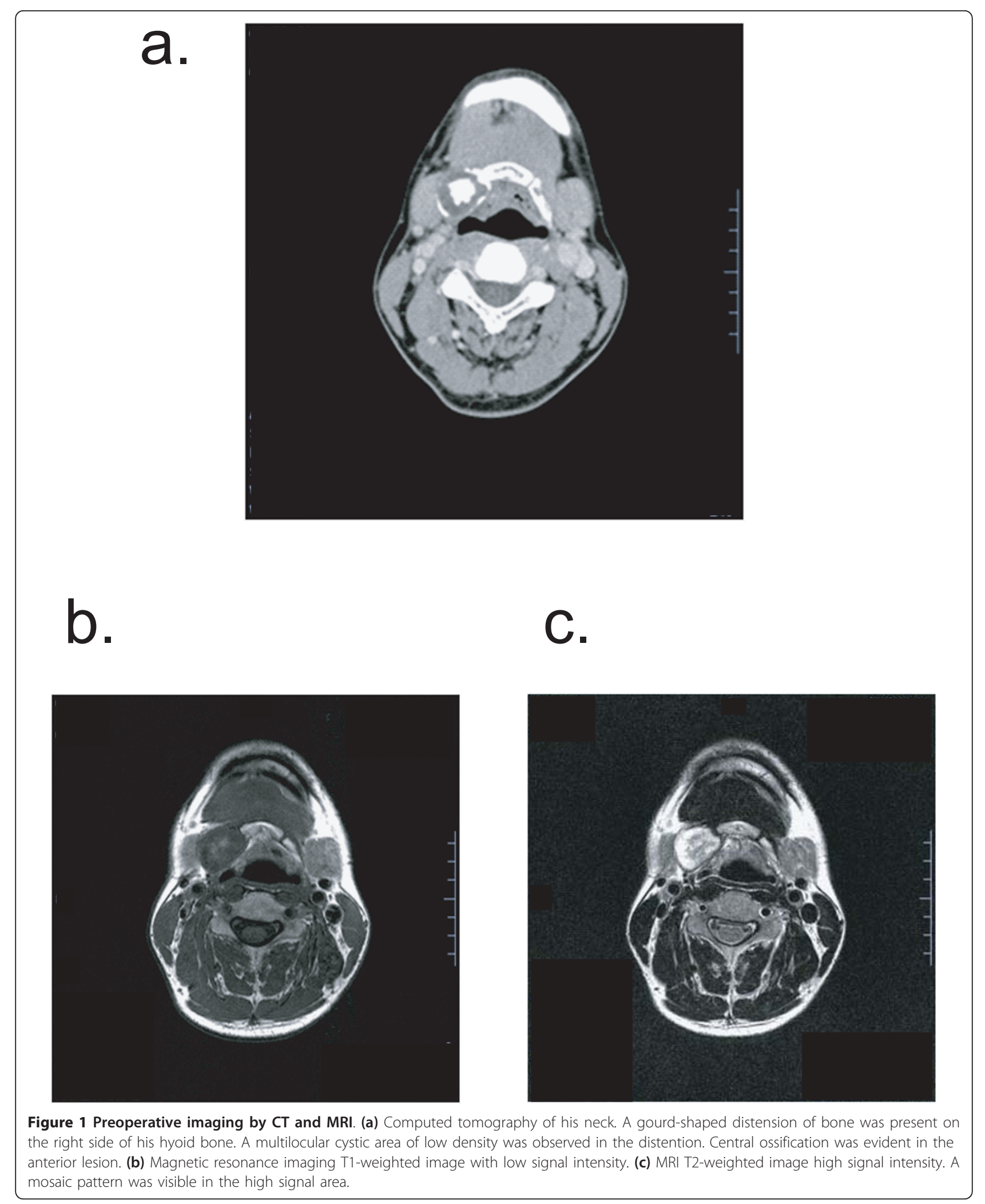




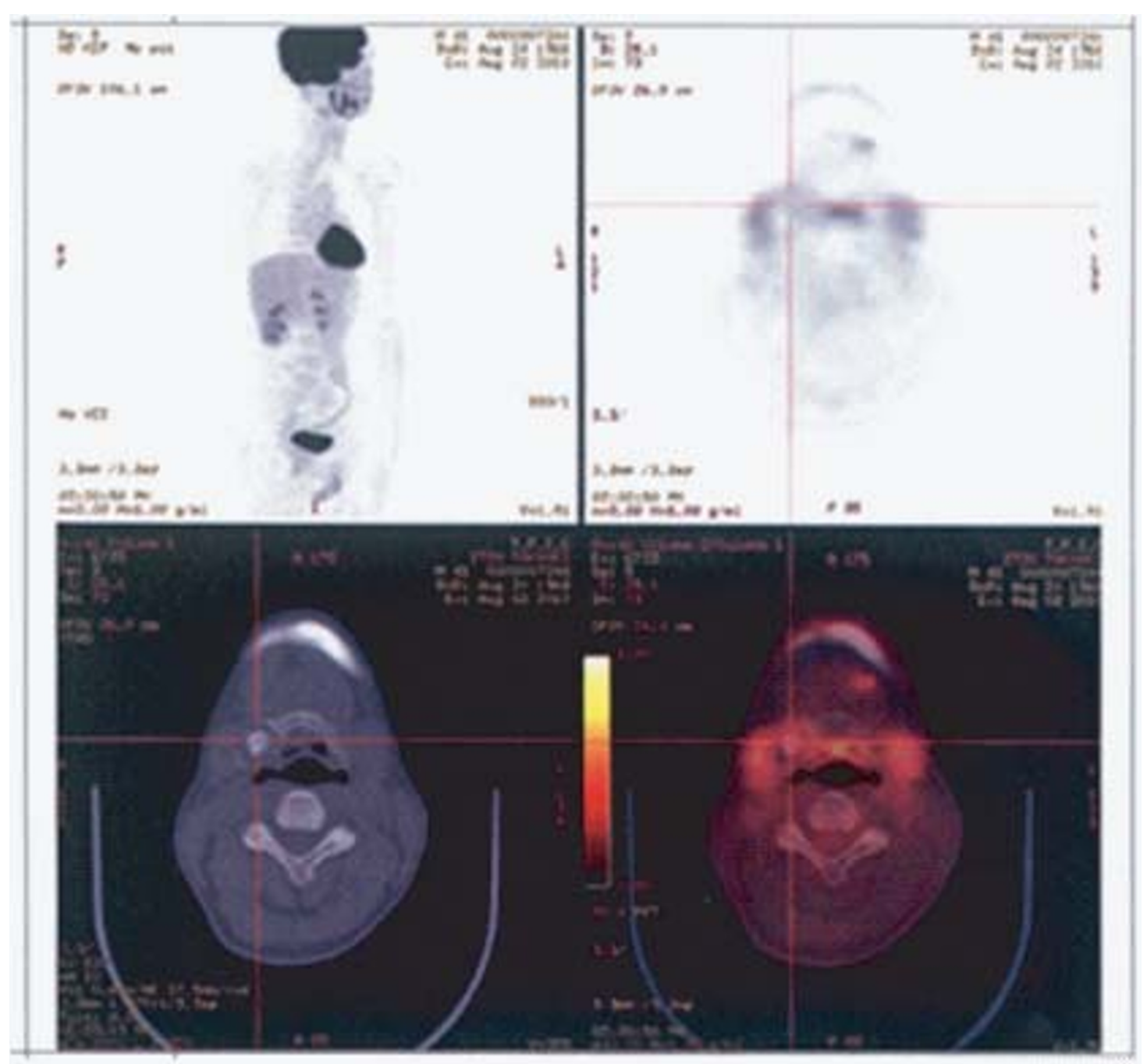

Figure 2 Positron emission tomography-computed tomography. No abnormal accumulation in the hyoid tumor or systemic abnormalities were evident.

An analysis of his blood samples revealed no contributory abnormalities.

Surgery was performed to extirpate the tumor. His geniohyoid, mylohyoid, sternohyoid, and thyrohyoid muscles were detached from his hyoid bone. The tumor mass and surgical margins were resected bilaterally on the left side of the body of his hyoid bone and the right greater horn. There were almost no adhesions between the tumor and the tissue around it. Following a favorable postoperative course, our patient was discharged from hospital.

A macroscopic examination identified the cartilaginous tumor as a semi-lobular mass covered by a thin fibrous coat. Histopathological examination revealed that it was relatively well differentiated, but with a somewhat high cell density and many binuclear cells. An open chromatin pattern was evident and intranuclear structures were observed in many cells. The condition was diagnosed as chondrosarcoma, grade one (Figure $3 \mathrm{a}-\mathrm{c}$ ). No additional surgical treatment was required. Our patient has been periodically followed up at our outpatient clinic. Twelve months postoperatively, the clinical course has been favorable and no local recurrences or remote metastases have been detected.

\section{Discussion}

Generally, the most useful diagnostic imaging examination for chondrosarcoma is $\mathrm{CT}$, which detects irregular bone destruction. Intratumoral calcification is noted in about $75 \%$ of patients using this tool [2,3]. MRI is useful for surgical planning and for evaluating the degree of invasion of the tumor into the surrounding soft tissues. Signal intensity is low in T1-weighted images and high in T2-weighted images; the calcified portion is signal- 
a.

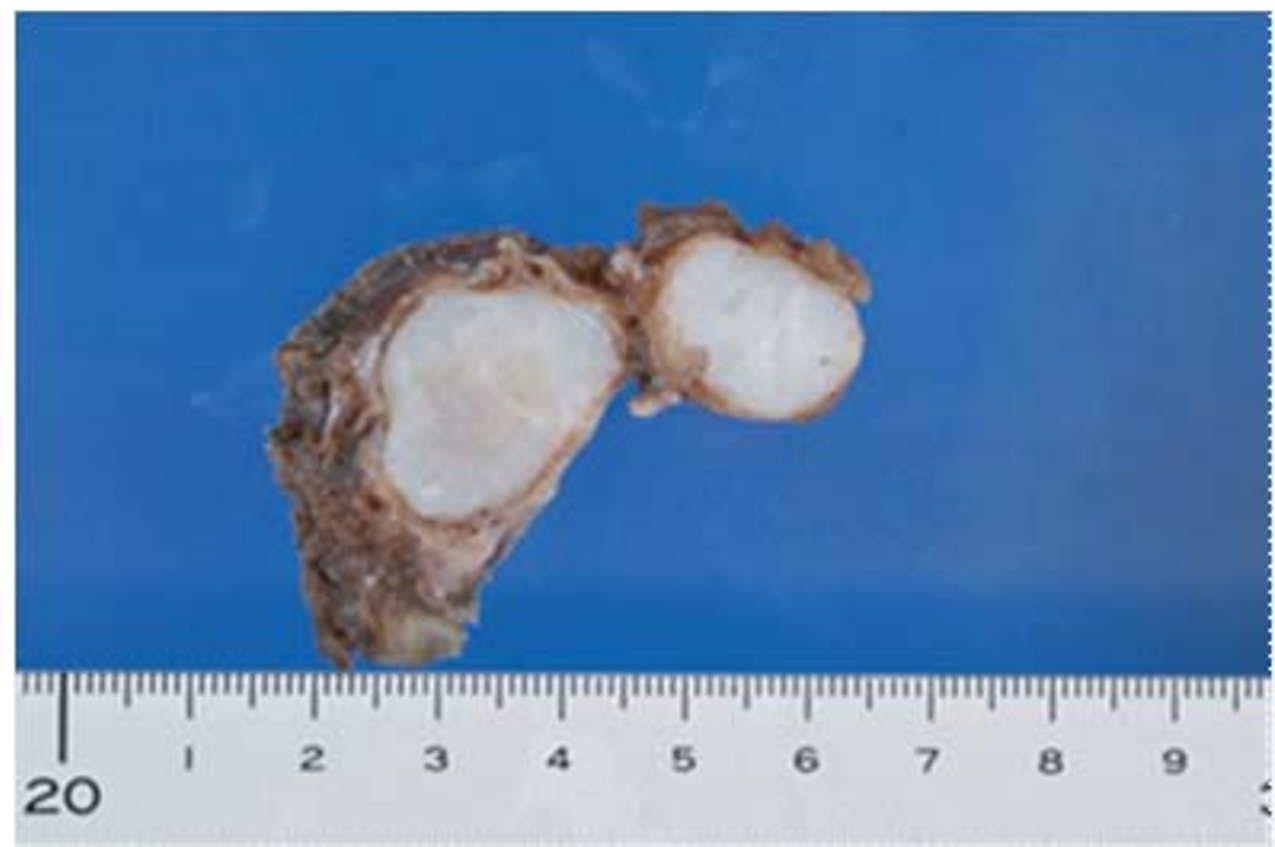

b.

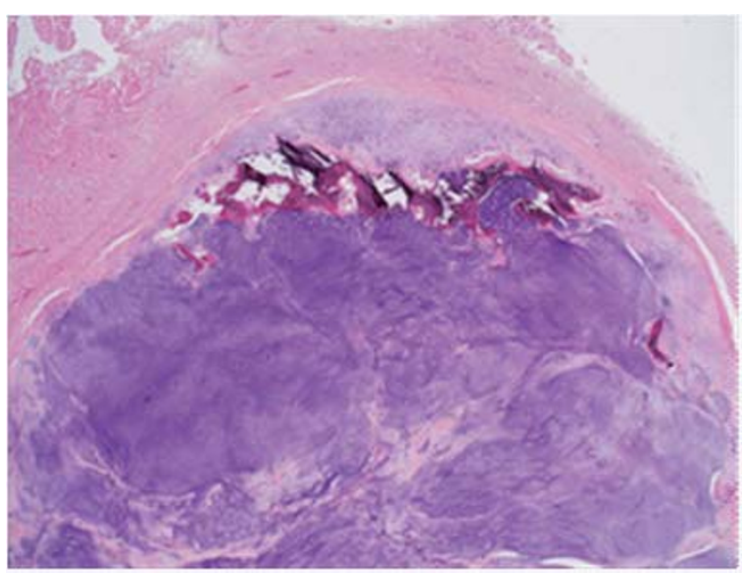

C.

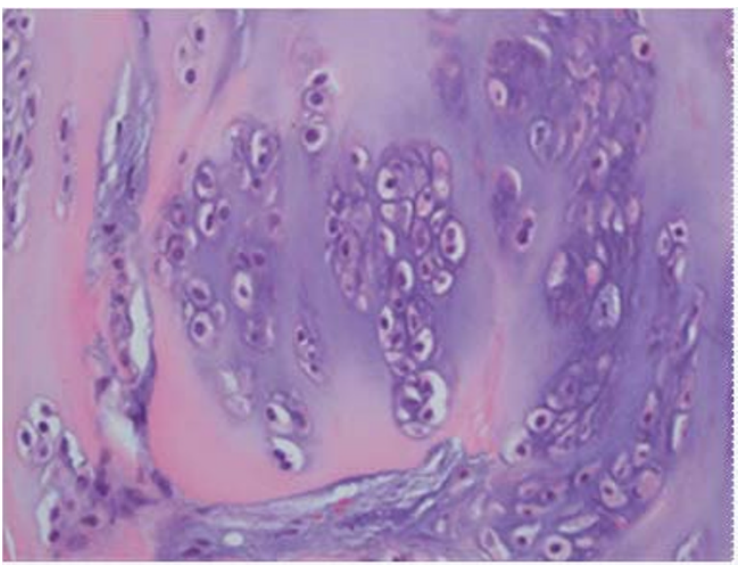

Figure 3 Macroscopic and histopathological examination. (a) The extirpated specimen was revealed to be a semi-lobular cartilaginous tumor mass covered by a thin fibrous coat. (b,c) Histopathological findings: (b) hematoxylin-eosin (HE)-stained, $\times 2$; (c) HE-stained, $\times 40$. The cartilaginous tumor mass is relatively well differentiated, the cell density is somewhat high and many binuclear cells were seen; an open chromatin pattern is evident with intranuclear structures observable in many cells.

free. A characteristic finding is a mosaic pattern in high signal areas in T2-weighted images [4]. In our case, a $\mathrm{CT}$ examination demonstrated no evidence of bone destruction, but revealed intratumoral calcification. On MRI examination, the signal intensity was low in T1weighted images and high in T2-weighted images, with 
a mosaic pattern; however, no invasion into the tissue around the lesion was seen.

In most cases, bone scintigraphy examination reveals poor uptake in the chondrosarcoma itself and morbid uptake in the destroyed area of the affected bone [5]. FDG-PET is useful for grading tumors and for evaluating local recurrences and metastatic diseases [6]. A standardized uptake value of 1.3 has been reported as a border value that distinguishes between benign and malignant tumors [7]. However, bone scintigraphy and FDG-PET did not rule out the presence of chondrosarcoma in our case. The negative test results may have been obtained partly because the hyoid bone itself is small, and partly because the reactive bone formation was inconspicuous on histology. Therefore, it may be impossible to distinguish chondroma from low-grade chondrosarcoma of hyoid origin by means of preoperative imaging tests, including positron emission tomography-computed tomography (PET-CT).

Generally, the occurrence of metastases becomes more probable as the degree of malignancy increases, resulting in poor prognoses [8]. The five- year survival rate for all cases of chondrosarcoma is reportedly $90 \%$ for grade one, $81 \%$ for grade two and $43 \%$ for grade three [9]. However, in the few grade one cases of hyoid origin with available outcome records, local recurrences occurred in five of the 10 reported cases. Therefore, close follow-up is necessary.

\section{Conclusion}

The present case did not involve union of the lesion to the tissue around it. The tumor and surrounding intact hyoid bone tissue were completely resected. Pathological results led to the diagnosis of a grade one tumor of low malignancy. Even though our radiographic studies, including PET-CT, were inconclusive, we surgically removed the cartilaginous tumor en bloc and the tumor was diagnosed based on the results of pathological investigations. Chondrosarcomas of hyoid origin have a high incidence of local recurrence and are sometimes metastatic. Therefore, it seems necessary to closely follow-up the patient for a prolonged period of time.

\section{Consent}

Written informed consent was obtained from the patient for publication of this case report and any accompanying images. A copy of the written consent is available for review by the Editor-in-Chief of this journal.

\section{Authors' contributions}

HT was involved in drafting the manuscript and reviewing the literature. HF was a major contributor in revising the manuscript and getting informed consent from our patient. MI was involved in reviewing the literature. YW reviewed the manuscript. All authors were responsible for the diagnosis, treatment and follow-up of the patient described in this case report. All authors read and approved the final manuscript.

\section{Competing interests}

The authors declare that they have no competing interests.

Received: 25 September 2011 Accepted: 18 January 2012

Published: 18 January 2012

\section{References}

1. Koch BB, Karnell LH, Hoffman HT, Apostolakis LW, Robinson RA, Zhen W, Menck HR: National cancer database report on chondrosarcoma of the head and neck. Head Neck 2000, 22(4):408-425.

2. Demeyere A, De Somer F, Perdieus D, Van Den Hauwe L, Lemmens L, Schillebeeckx J: Chondrosarcoma of the hyoid bone. Eur Radiol 2000, 10(2):308-309.

3. Hediger R, McEniff N, Karmody C, Eustace S: Recurrent chondrosarcoma of the hyoid bone. Clin Imaging 1997, 21(1):69-72.

4. Itoh K, Nobori T, Fukuda K, Furuta S, Ohyama M: Chondrosarcoma of the hyoid bone. J Laryngol Otol 1993, 107(7):642-646.

5. Greer JA Jr, Devine KD, Dahlin DC: Gardner's syndrome and chondrosarcoma of the hyoid bone. Arch Otolaryngol 1977, 103(7):425-427.

6. Umekita Y, Yoshida A, Yoshida H: Parosteal chondrosarcoma attached to the hyoid bone: A case report. Int J Oral Maxillofac Surg 1998, 27(3):215-216.

7. Aoki J, Watanabe H, Shinozaki T, Tokunaga M, Inoue T, Endo K: FDG-PET in differential diagnosis and grading of chondrosarcomas. J Comput Assist Tomogr 1999, 23(4):603-608.

8. Bough ID Jr, Chiles PJ, Fratalli MA, Vernose G: Laryngeal chondrosarcoma: two unusual cases. Am J Otolaryngol 1995, 16(2):126-131.

9. Evans HL, Ayala AG, Romsdahl MM: Prognostic factors in chondrosarcoma of bone: a clinicopathologic analysis with emphasis on histologic grading. . Cancer 1977, 40(2):818-831.

doi:10.1186/1752-1947-6-21

Cite this article as: Tachino et al: A low-grade chondrosarcoma presenting as an unusual cervical mass in the hyoid bone: a case report. Journal of Medical Case Reports 2012 6:21.

\section{Submit your next manuscript to BioMed Central and take full advantage of:}

- Convenient online submission

- Thorough peer review

- No space constraints or color figure charges

- Immediate publication on acceptance

- Inclusion in PubMed, CAS, Scopus and Google Scholar

- Research which is freely available for redistribution 